\title{
VISUALIZATION OF THE BATTLESPACE: A CORNERSTONE OF MODELING FOR ANTICIPATORY BEHAVIOR
}

\author{
Denise L. Aleva \\ Janet E. Miller \\ Human Effectiveness Directorate \\ Air Force Research Laboratory \\ 2255 H Street \\ Wright-Patterson Air Force Base OH, 45433-7022, U.S.A.
}

\begin{abstract}
In order to achieve Predictive Battlespace Awareness, representations of the battlespace must be accurate and timely. They must also provide predictive and actionable information to the user. Planners must be able to predict the impact of friendly operations on the enemy in order to stay inside his decision cycle. Further, they must be able to dynamically adapt an effects-based plan based on assessment of operations and the campaign. This paper outlines an operator-centered program for developing the models needed to provide the information and a systems engineering approach to designing visualizations which convey the information output to decision makers. Fundamental to the process is warfighter involvement at all phases.
\end{abstract}

\section{INTRODUCTION}

With the changing character of warfare, information superiority is a high priority. More than ever before, coordination, synchronization, and interoperability among our own forces and with our allies requires that everyone involved share a common knowledge of the battlespace to enable a coherent combined air, land, and maritime force operations.

Conflicts no longer take place purely in the physical domain, although the physical domain is where the military seeks to influence exists. Alberts, Garstka, Hayes and Signori (2001) describe two other domains of warfare. The information domain is where information is created, manipulated, stored and shared. The command and control of modern military forces is communicated, where commander's intent is conveyed. The cognitive domain is where, as a result of sensemaking, decisions are made. These decisions are influenced by perceptions, awareness and understanding as well as beliefs and values.

Given the complexity of current and future operating environments, strategy planners need a multidimensional understanding of the battlespace. Asymmetric warfare necessitates that our strategists look beyond targets-based operations, where we simply identify and destroy enemy entities. Effects based operations models the enemy as a system which reacts to our actions. This requires the capability to predict the adversary response to a selected action. Actions may be diplomatic, information, military or economic (DIME). Effects may be political, military, economic, social, information or infrastructure (PMESII). Timing must be explicitly considered and effects dynamically assessed.

Effects based operations leads to far more possible combinations of actions than target-based or other operations which then requires that the decision maker be fully informed on PMESII aspects. Given the complexity of decision making involved, information systems must have the flexibility and capability to deal with ambiguous operational problems and tasks, new types of emergent threats and opportunities, and a broad set of operational stakeholders and perspectives (Leedom, 2003).

In order to achieve Predictive Battlespace Awareness, representations of the battlespace must be accurate and timely. They must also provide predictive and actionable information to the user. Planners must be able to predict the impact of friendly operations on the enemy in order to stay inside his decision cycle. Planners must be able to dynamically adapt an effects-based plan based on assessment of operations and the campaign. In addition to achieving the desired effects, they must guard against undesired or unpredicted interactions. With advanced visualization, we can better manage the attention of the decision maker to all the pertinent information.

This paper describes an operator-centered program, Centers of Gravity (COG) Visualization, for enhancing the commanders' understanding of the battlespace and allowing them to proactively plan friendly operations in a more efficient and effective way. The program is a joint effort 


\section{Aleva and Miller}

between Air Force Research Laboratory's (AFRL) Human Effectiveness (HE) and Information (IF) Directorates.

Section 2 of the paper outlines the requirements which this program addresses. Section 3 describes the Commanders' Predictive Environment program and its objectives. Section 4 specifically addresses the visualization requirement and the systems engineering approach we are applying to develop the visualizations. Finally, in Section 5, we present our conclusions related to the problem of achieving predictive battlespace awareness.

\section{BACKGROUND AND REQUIREMENTS}

Joint Vision 2020 emphasizes the importance of information superiority to support the strategic concepts of decisive force, power projection, overseas presence and strategic agility. Joint Publication 1-02 defines information superiority as the capability to collect, process, and disseminate an uninterrupted flow of information while exploiting or denying an adversary's ability to do the same. However, JV2020 goes on to emphasize that information superiority provides the joint force a competitive advantage only when it is effectively translated into superior knowledge and decisions. Joint Publication 1 calls for a common intelligence picture as an enduring enabler.

Information superiority can only be achieved by addressing the challenges of data/information overload. As stated in Patterson, Roth and Woods (1999), "Common to most implicit definitions of data overload....is the notion that somehow an excessive amount of data creates additional cognitive burdens for the human operator." Joint warfighters do not have the resources to review and understand all relevant data to a problem, so different strategies must be employed, either consciously or unconsciously, to avoid or cope with data overload. One would be limiting the input source. For example, an individual might decide he only wants to gather data from one source. An intelligence analyst (IA) might decide that he will not review tertiary sources. Another would be bounding the data set. The IA may decide he will only pay attention to technical exchanges between his country of interest and particular other countries or only look at documents after a certain date. But a third way which is being chosen more often is relying on automation to offload the task (Woods et al, 1996), especially through the use of visualization. From CIA's P1000 strategy document (P1000 Visualization Planning Committee 1996): "Successful information visualization will enable analysts to cope with the current information overload and will fundamentally change how they allocate their time in the analysis process."

Over the past decade or so, many have warned of the increasing data/information overload challenges caused by the implementation of advanced information technology within the military. In fact, today's warfighter is indeed having the foretold challenges. Effective visualization can help address data/information overload and can support information superiority because it is a natural way for a human to take in a lot of information in a natural way. As stated in Woods (1994), "When observers scan a visual scene or display, they tend to look at 'informative' areas". Informativeness, defined as some relation between the viewer and scene, is an important determinant of eye movement patterns." Therefore, care must be taken in forming the visualizations to ensure uncluttered views, smooth work processes and the user's ability to find significance in the data presented. With understanding of the work to be performed to give the proper context during the development of visualizations, they will support information superiority.

In December, 2005 U. S. Joint Forces Command held a Visualization Industry Day, requesting the help of industry and academia to deliver advanced visualization technologies to the joint warfighting community. The audience was told that joint force commanders want sophisticated three-dimensional visualization that displays geospatial data for all the elements of national power, including political military, economic, social, infrastructure and information. Coming up with satisfactory solutions for the warfighting community is a goal of the AFRL programs described below.

\section{COMMANDERS PREDICTIVE ENVIRONMENT}

The goal of the joint AFRL HE and IF Commander's Predictive Environment (CPE) program is to enhance the Joint Forces Commander (JFC), Joint Forces Air Component Commander (JFACC) and senior commanders' decision making process by supporting their ability to envision future battlespace options. CPE will create technologies that support the JFC/JFACC and senior commanders' understanding of events and factors affecting adversaries, neutrals, and self over time so that action can be taken to shape the battlespace when possible. CPE will enhance the commanders' understanding of the battlespace and allow them to proactively plan friendly operations in a more efficient and effective way.

\section{Capability 1. Understand the battlespace}

Understand the basic information and the interactions among PMESII impacts on self (and allies), adversaries, or neutrals, in the time epochs of past, present and the future.

\section{Capability 2. Evaluate courses of action (COAs)}

Understand how our COAs alter plausible futures to achieve the commander's intent. Our COAs are expressed in terms of DIME actions that we may take. 


\section{Aleva and Miller}

\section{Capability 3. Access and share battlespace Information}

This function can best be described in terms of four objectives that center around the sender, the recipient, and the media used to gather, store, process, display, and disseminate CPE-related information:

Objective 1: Identify and access critical information relevant to pending decisions.

This includes identifying and accessing relevant data for context-dependent critical information relevant to pending decisions. CPE should provide the capability to perform filtered searches that be accessed according to criticality of information (e.g., routine, critical). CPE should provide the data that can reveal critical nodes and vulnerabilities that may be used in effects-based operations. This information will allow commanders and staff to present possible actions and anticipated consequences to others that need the information and to integrate the information with other relevant systems.

Objective 2: Perceive data with multiple sensory modes.

This objective recognizes that "visualizing" the battlespace by itself is too limiting. CPE should allow users to perceive cognitive bias-free data using multiple sensory modes and varying degrees of resolution as required for communication. It addresses alternative information processing functions, varying cognitive levels, styles, or function, and considers framing and other well-defined cognitive biases

Objective 3: Allow data sharing to be tailored to the style of communicator and recipients.

This objective recognizes that when it comes to sharing and communicating information, one size doesn't fit all. Decision environments that force the user to operate the way the environment dictates rather than being adaptable to the preferences of the user may be destined to failure. This incorporates the notion that within CPE, communication (and decision making) aids should be "personalized" and tailorable to allow different users to operate in the environment in different ways

Objective 4: Communicate and share high quality, understandable, and credible information

This final objective states CPE must provide the user with the ability to communicate and share high quality, credible information in a usable, timely, understandable, useful, and defensible way. Dissemination of information may be available either through information push or pull capabilities. CPE needs the capability to play forward or back the operational situation, to visualize, compare and contrast multiple future states and implications, and to share assessments and rationale with peers, superiors, and subordinates. It must display the current battlespace situation, communicate selected options and commanders' intent, and facilitate collaboration with other decision makers.

While all three of the capability areas define CPE in general, visualization is the cornerstone to modeling for anticipation for the CPE program. In addition, visualization is a research area that brings together some of the strengths of both information technology and human effectiveness, and warrants multi-disciplinary collaboration between researchers from the AFRL IF and HE Directorates.

\section{VISUALIZATION}

In order to achieve the objectives of the CPE program, it is imperative that we provide the users with Decision Quality Information while not overwhelming them with more data. Decision makers need to be aware of the uncertainties associated with the information they are using. They need to look backward in time to examine trends and forward in time to assess the effects of possible actions. Decision makers need Visualization of alternative hypothesis or alternative futures. They need to be able to run "What if" scenarios, examining possible adversary responses to potential courses of action. This requires the display of fourdimensional information, three spatial dimensions plus the capability to look backward and forward in the time dimension.

\subsection{Centers of Gravity Visualization}

The Center of Gravity (COG) Visualization project is intended to facilitate predictive battlespace awareness through visualizing adversary and friendly centers of gravity in order to support mission planning decision-making prior to and during execution. Our Department of Defense defines Center of Gravity as "those characteristics, capabilities or sources of power from which a military force derives its freedom of action, physical strength or will to fight."

Visualization of intelligence information referred to as Centers of Gravity or Warden's Rings will promote full understanding of all aspects of adversary strengths and weaknesses. Visualization of Centers of Gravity will provide the extensive data about adversary forces, organic essentials, infrastructure, leadership, population, and science and technology in an easily accessible and understandable format. This will enhance Information Operations, and therefore the capability to predict and counter adversary courses of action.

Our COG visualizations are graphical representations that assist the analyst/planner to rapidly and accurately identify the appropriate COGs and employ analytical 


\section{Aleva and Miller}

methods to develop, compare and recommend methods or courses of action to exploit these COGs. These graphical representations will also assist the commander to understand the significance of and effectively exploit COGs.

\subsection{Visualization Approach}

Our approach to developing the COG visualizations is depicted graphically in Figure 1. In order to determine what information was needed to perform a centers of gravity analysis, we implemented a multi-path analytical approach. This approach follows the Department of Defense (DoD) Architectural Framework (DoDAF), Version 1.0, which defines a common approach for DoD architecture description development. We conducted a literature search of the Operational requirements and acquired training materials from the $505^{\text {th }}$ Training Squadron at Hurlburt Field.

We used concept mapping software to graphically depict the Intelligence Preparation of the Battlefield procedure; thus mapping doctrine to operations and process to objectives. Concept mapping (McNeese, Zaff, Citera, Brown and Whitaker, 1995; Thordsen, 1991) is a method that engages the expert with the domain investigator while an actual map of the concepts and their interrelationships is drawn. Concepts, which can be actions, events or objects, are nodes with labeled, directional links between nodes. Having an artifact for the expert and the domain investigator to view during discussion supports communication and understanding. This concept map, while not required as part of the DoDAF documentation, provided a way of visualizing the IPB process. Traceability of the processes to the various requirements documents was maintained throughout the building of the IPB process model. Each element of the concept map was linked to the related paragraphs of the requirements documents. We held discussions with subject matter experts from the intelligence community to gain further insight into the Centers of Gravity analysis process and the associated information requirements. Use cases were then developed from the models. A use case is a detailed technology-free description of what must be accomplished from the perspective of the analyst. We then visualized the use cases in sequence diagrams, showing the sequence of actions, who performs the actions and their relationships and interactions.

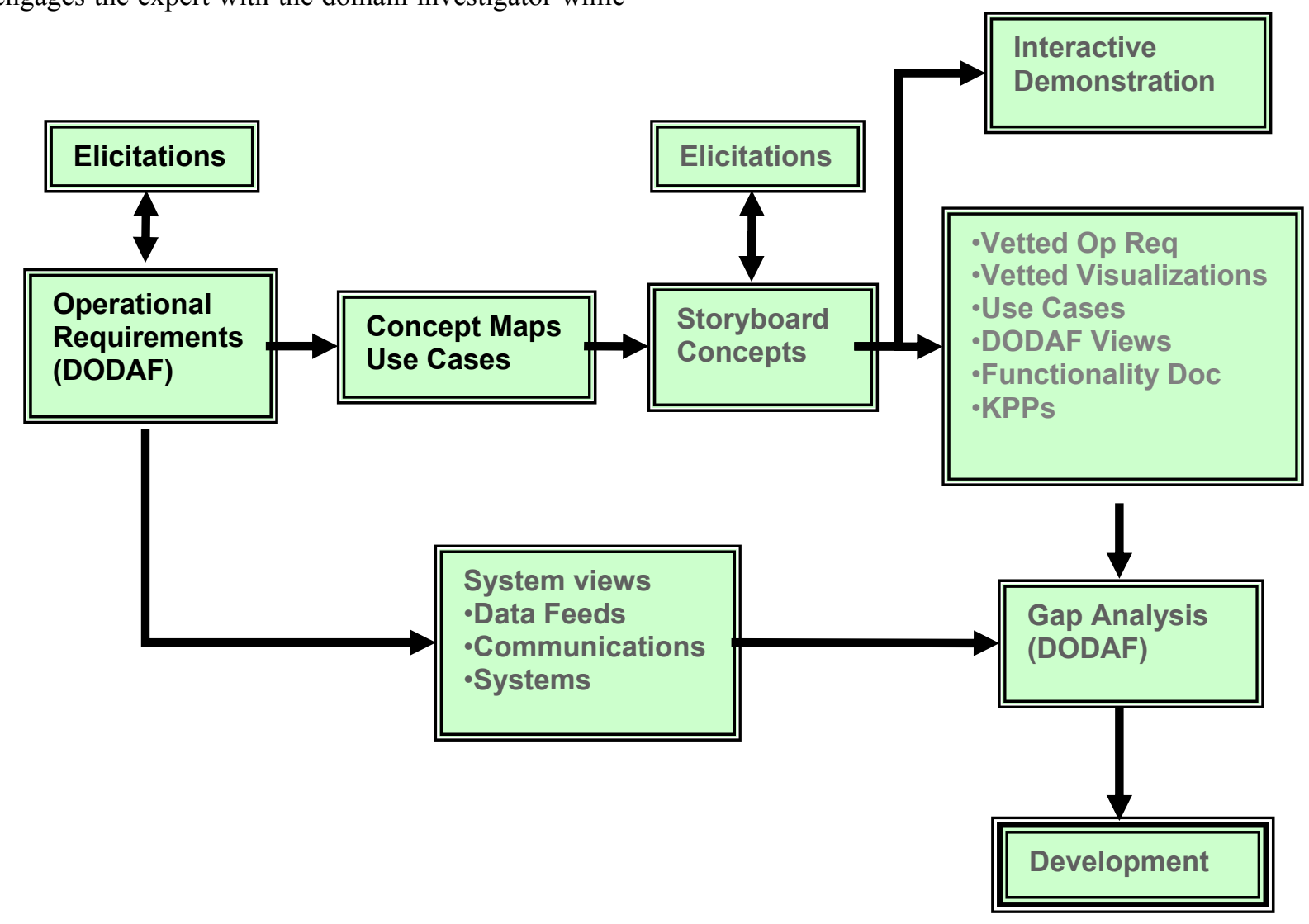

Figure 1: Process Flow for Center of Gravity Visualization Development. 
Although Joint Publication 1-02 defines centers of gravity as "those characteristics, capabilities, or localities from which a military force derives its freedom of action, physical strength, or will to fight" there are a number of different models that define these "characteristics, capabilities, or localities." In order to integrate the various COG analysis concepts found in the literature, we found it necessary to develop an ontology that relates the terminology used in the various models.

Information requirements analysis for decision support was conducted using the information from the models. Visualization requirements were derived from this analysis.

We developed visualization concepts in close collaboration with the SMEs and eventual users. Storyboard drawings were created during elicitation sessions and rapid prototyping software allowed these concepts to be quickly inplemented for further vetting with the SMEs. User assessment and demo updates were thus accomplished by an iterative process.

\section{CONCLUSIONS}

Decision makers must create and maintain complicated mental models of the battlespace. While some information about the physical battlespace can be depicted on a twodimensional geospatial display, many elements of centers of gravity do not lend themselves to geospatial display and are provided to the decision maker as text files or tables. In addition, the relationships and interactions among the various centers of gravity are not easily understood.

Decision makers may need to weigh information against several alternative plausible futures in order to evaluate courses of action. Modeling and simulation and improved visualization of complex model output is needed to facilitate predictive battlespace awareness and achieve decision superiority, to support advanced command and control capabilities, and to reach the full potential of dominant maneuver, precision engagement, full dimensional protection and focused logistics. The breadth and pace of this evolution requires flexibility and a willingness to innovate.

Visualization of intelligence information referred to as Centers of Gravity or Warden's Rings is needed to promote full understanding of all aspects of adversary strengths and weaknesses. Visualization of Centers of Gravity will provide the extensive data about adversary forces, organic essentials, infrastructure, leadership, population, and science and technology in an easily accessible and understandable format. This will enhance Information Operations, and therefore the capability to predict and counter adversary courses of action.

The development of advanced visualizations for decision superiority requires a highly structured approach and a multidisciplinary team. User participation at all phases is of the utmost importance.

\section{DISCLAIMER}

The views expressed in this paper are those of the authors and do not reflect the official policy or position of the U.S. Air Force, Department of Defense, or the United States. Government.

\section{REFERENCES}

Alberts, D. S., J. J. Garstka, R. E. Hayes, and D. A. Signori. 2001. Understanding Information Age Warfare. CCRP Publication Series: 77.

Joint Vision 2020. 2000. U. S. Government Printing Office. Washington D.C.

Joint Publication 1. 2000. Joint Warfare of the Armed Forces of the United States. Directorate for Operational Plans and Joint Force Development, United States Joint Forces Command Joint Warfighting Center, Suffolk, Virginia.

Joint Publication 1-02. 2000. Department of Defense Dictionary of Military and Associated Terms. Directorate for Operational Plans and Joint Force Development, United States Joint Forces Command Joint Warfighting Center, Suffolk, Virginia.

Leedom, D. K. 2003. Functional analysis of the next generation common operating picture. Proceedings of the $8^{\text {th }}$ annual International Command and Control Research and Technology Symposium. Washington D.C

McNeese, M. D., B. S. Zaff, M. Citera, C. E. Brown, and R. Whitaker. 1995. AKADAM: Eliciting user knowledge to support participatory ergonomics. International Journal of Industrial Ergonomics. Vol 15, 345363.

Woods, D.D. 1994. Visual Momentum: A concept to improve the cognitive coupling of person and computer. International Journal of Man-Machine Studies. Vol 21, 229-244.

Woods, D. D., E. S. Patterson, J. M. Corban, and J. C. Watts. 1996. Bridging the Gap between User-Centered Intentions and Actual Design Practice. Human Factors and Ergonomics Society 40th Annual Meeting. Philadelphia, PA.

Woods, D.D., E. S. Patterson, and E. M. Roth. 1999. Aiding the Intelligence Analyst in Situations of Data Overload: A Simulation Study of Computer-Supported Inferential Analysis Under Data Overload. Institute for Ergonomics/Cognitive Systems Engineering Laboratory Report, ERGO-CSEL 99-02, The Ohio State University, Columbus $\mathrm{OH}$. 


\section{AUTHOR BIOGRAPHIES}

DENISE L. ALEVA is an Engineering Research Psychologist at the Human Effectiveness Division of Air Force Research Laboratory. She earned a B.S. in Psychology and a M. A. in Applied Behavioral Science from Wright State University and a M. S. in Electro Optics from the University of Dayton. She is currently directing research in the area of Battlespace Visualization, particularly visualizations for Air Operations Centers.

JANET E. MILLER is an Engineering Research Scientist with an emphasis in decision sciences at the Air Force Research Laboratory, Human Effectiveness Directorate, Wright Patterson AFB, Dayton, OH. Her experience includes gathering user requirements and implementing automated systems at the Pacific Air Forces and the National Air and Space Intelligence Center. Her degrees include a BS in Electrical Engineering from Louisiana Technical University, an MS in Management of Information Systems from the University of Arizona and a Ph. D. in Cognitive Systems Engineering from the Ohio State University. Additionally, she is holds the rank of Lieutenant Colonel in the US Air Force Reserves (Active). 